to policy they have been amply justified by experience. Yet, in these times of economic stringency, the policy-makers may wish to know whether, for example, the provision of a particular vitamin concentrate to pregnant women can safely be discontinued. Survey data will not provide an answer except in terms of current intake levels and standards. A more precise answer must await the acquisition of more detailed physiological knowledge, or must be obtained ad hoc by means of a carefully controlled feeding experiment-an undertaking even more difficult than a field survey.

The Aberdeen inquiry was partly supported by grants from the Secretary of State for Scotland, on the recommendation of the Advisory Committee on Medical Research. I wish to express the gratitude of the research team.

\title{
REFERENCES
}

Antonov, A. N. (1947). F. Pediat. 30, $25^{\circ}$.

Baird, D. (1952). Amer. F. Obstet. Gynec. 63, 1200.

Baird, D. \& Thomson, A. M. (1954). Gynaecologia, 138, 266.

Baird, D., Walker, J. \& Thomson, A. M. (1954). F. Obstet. Gynaec., Brit. Emp., 6r, 433.

Bernard, R. M. (1952). Edinb. Med. F. 59, Proc. Edinb. Obstet. Soc. p. I.

Boyne, A. W. \& Leitch, I. (I954). Nutr. Abstr. Rev. 24, 255.

Chesley, L. C. (1944). Amer. F. Obstet. Gynec. 48, 565.

Duncan, E. H. L., Baird, D. \& Thomson, A. M. (I952). F. Obstet. Gynaec., Brit. Emp., 59, 183.

Garry, R. C. \& Wood, H. O. (1945-6). Nutr. Abstr. Rev. 15, 59x.

Hobson, W. (1948). F. Hyg., Camb., 46, 198.

Hytten, F. E. (I95 I). Arch. Dis. Child. 26, 477.

Hytten, F. E. (1954). Brit. med. Y. ii, 844.

Hytten, F. E. \& Thomson, A. M. (1955). Brit. med. F. ii, 232.

Illsley, R. (1955). Brit. med.F. ii, 1520.

Leitch, I. (1957). Proc. Nutr. Soc. 16, 38.

Leitch, I. \& Aitken, F. C. (I949-50). Nutr. Abstr. Rev. 19, 507.

Macy, I. G., Moyer, E. Z., Kelly, J. H., Mack, H. C., Di Loreto, P. C. \& Pratt, J. P. (1954). F. Nutr. 52, Suppl. x.

McGanity, W. J., Bridgforth, E. B., Martin, M. P., Newbill, J. A. \& Darby, W. J. (1955). 7. Amer. diet. Ass. 3I, 582 .

Smith, C. A. (1947). F. Pediat. 30, 229.

Sontag, L. W. \& Wines, J. (r947). Amer. J. Obstet. Gynec. 54, 994.

Thomson, A. M. (I95 I). Brit. $\mathcal{F} . N u t r .5$, 58 .

Thomsan, A. M. \& Duncan, D. L. (1954). Nutr. Abstr. Rev. 24, x.

Thomson, A. M. \& Billewicz, W. Z. (1955). Proc. Nutr. Soc. 14, v.

Thomson, A. M. \& Billewicz, W. Z. (1957). Brit. med. $\mathcal{~ . ~ ( I n ~ t h e ~ P r e s s . ) ~}$

Yorston, J., Hytten, F. E. \& Thomson, A. M. (1957). In preparation.

\section{Placental transmission and foetal nutrition}

\author{
By E. C. Amoroso, Royal Veterinary College and Hospital, London
}

Text for publication not received. 